\title{
ANALYTICAL INVESTIGATION OF SELECTED PESTICIDE RESIDUES FROM FRUITS AND VEGETABLES BY AN IMPROVED EXTRACTION METHOD USING REVERSE PHASE HIGH PERFORMANCE LIQUID CHROMATOGRAPHY

\author{
Sadia Ata, 1 *Feroza Hamid Wattoo, ${ }^{2}$ Momina Feroz, ${ }^{1}$ Muhammad Hamid Sarwar Wattoo, ${ }^{3}$ \\ Syed Ahmed Tirmizi, ${ }^{3}$ Muhammad Javaid Asad ${ }^{2}$ \\ http://dx.doi.org/10.4314/ejesm.v6i4.1
}

Received 23rd September 2012; accepted 25th June 2013

\begin{abstract}
Pesticide residues like chlorpyrifos, cypermethrin, deltamethrin and dichlorvos were monitored by an improved extraction method from apple, peach and potato using reverse phase high performance liquid chromatography. The selected pesticides were extracted from fruits and vegetable samples using smaller volume of ethyl-acetate, anhydrous sodium sulphate, and sodium chloride. Cleanup of extract was done with activated charcoa. Final extracts of fruits and vegetable samples were analysed using reverse phase high performance liquid chromatography with a ultra-violet detector in an isocratic mode. Maximum residue levels (MRL), 0.924, 1.630 and $0.454 \mu \mathrm{g} / \mathrm{g}$ for chlorpyrifos, cypermethrin and deltamethrin were found in peach respectively. The MRL $0.283 \mu \mathrm{g} / \mathrm{g}$ for dichlorvos was found in apple samples. All these observed results were compared with Codex maximum residue levels. It was found that cypermethrin and chlorpyrifos residual amounts were greater than MRLs, so their spray on the target crops should be limited because these are resistant to the degradation.
\end{abstract}

Keywords: Pesticide residues, reverse phase high performance liquid chromatography, chlorpyrifos, cypermethrin, deltamethrin, dichlorvos

\section{Introduction}

Pests are disease causing organisms that use plants as their source of food. The pests attack results in decreased yield of fruits and vegetables. Pesticides are agrochemicals formulated to combat the attack of pests on agricultural crops. In modern agricultural practices, pesticides are widely used on crops for pre and post harvest applications. Pesticides are classified based on target organisms, chemical structure, and physical state and are available in concentrated liquid or powder form. Various familiar classes of pesticides are herbicides, insecticides, rodenticides, biopesticides and weedicides (Cheng and Bersamira 1994; Gilden et al., 2010). A systematic pesticide follows absorption mechanism in plants. Pests are disease causing organisms that use plants as their source of food.

The pests attack results in decreased yield of fruits and vegetables. Pesticides are agrochemicals designed to combat the attack of pests on agricultural through the xylem and then outward to kill the plant attacking pests (American Chemical Society 2009).

Use of pesticides in Pakistan was started in 1952 (Tariq et al. 2007; Shams-ul-Alam and Awwal Ahmed 2009). The pesticide companies have now motivated the farmers to use more than the recommended dose of pesticides through the media campaign. At present, 108 types of insecticides, 30 types of fungicides, 39 types of weedicides, 5 types of acaricides are being used in Pakistan (Ambedkar et al., 1999; Tariq et al., 2007). It is estimated that $27 \%$ of the insecticides are being used on fruits and vegetables (Kmellara et al., 2008).

Pesticides cause potential toxicity to human beings and animals. Human health hazards vary with the extent of exposure. Moderate health hazards include flu, skin diseases blurred vision and headache. Severe human health hazards include paralysis, blindness and even death (Abhilash et al., 2009; Min et al., 2006).

According to Stockholm convention on persistent organic pollutants, 10 of the 12 most dangerous and persistent organic chemicals are the pesticides (Gilden et al., 2010; Miller et al., 2006). These synthetic pesticides are mostly persistent to degradation, may remain in fruits and vegetables and cause health hazards to the consumers.

Pesticides are analysed by simple gas chromatographic techniques, usually gas chromatography equipped with mass spectrometry (Min et al., 2006). In food samples, liquid chromatography hyphenated with tandem mass spectrometry proved to be a powerful 
technique for pesticide analysis (Ortelli et al., 2004). Analytical chemists' target is to develop cost-effective, robust and sensitive analytical technique that can identify and quantify the residual amounts of pesticides in food samples (Hernandez-Borges et al. 2009).

In general, the main criteria for sample preparation of pesticides residue analysis includes; multiresidue analysis, maximum recoveries, least matrix effects, good precision, and to develop easy and safe analytical method (Hernandez-Borges et al., 2009). High performance liquid chromatography HPLC, has also found its applications for pesticide analysis with ultraviolet and fluorescent properties. HPLC is used for a broad spectrum analysis because it can determine all ionic, polar, nonpolar, acidic, basic, neutral and thermally unstable pesticides (Siddique et al., 2003).

Reverse phase high performance liquid chromatography is good for separating and identification of organophosphorus and pyrethroids group of pesticides (Tariq et al., 2007; Hernandez-Borges et al., 2009). The objective of this research was to investigate pesticides residues in fruits and vegetable matrices with the improved extraction method by using smaller volumes of extracting solvents to make the analysis economical.

\section{Materials and Methods Chemicals and reagents}

HPLC grade purity solvents were purchased from MERCK and FLUKA. Anhydrous sodium sulfate, magnesium sulfate, activated charcoal and Florisil were of analytical grade. Stock and standard solutions of pesticides standards were prepared in acetonitrile and their retention time was recorded.

\section{Samples Collections}

Samples for fruits and vegetables like apple, peach and potato were purchased from the local market of Lahore city. One $\mathrm{kg}$ of each apple, peach and potato was collected for the laboratory analysis. Existing methods were improved by using smaller volume of extraction chemicals. Each sample of fruits and vegetable was chopped in an electrical chopper and $50 \mathrm{~g}$ of chopped sample was taken in an Erlenmeyer flask.

Sodium chloride ( $2.5 \mathrm{~g})$, anhydrous sodium sulfate $(10 \mathrm{~g})$ and ethyl acetate $(60 \mathrm{~mL})$ were added, homogenized with a horizontal shaker for an hour and filtered by an ordinary filter paper. Filtered extract was cleaned using $6 \mathrm{~g}$ of activated charcoal. The cleaned extract was concentrated on a rotary evaporator and was then dried by bubbling nitrogen gas through it. The dried extract was then redissolved in an acetonitrile solvent and final extract of one $\mathrm{mL}$ was ready for injection to liquid chromatograph.

\section{Analytical Techniques}

The methods for the pesticide residue analysis with conventional techniques are being improved day by day. The major steps involved in pesticide residue analysis are; extraction, cleanup and analysis. Therefore, reliable, costeffective multi-residue methods are required to monitor pesticide residues in food samples. Extraction of pesticide residues from fruits produces complex mixtures that have often required sample purification and preparation procedures to isolate the targeted pesticides for analysis.

The reverse phase high performance liquid chromatography was used as an analytical technique to get chromatograms showing the residual effects of each pesticide. The pesticide residue analysis was done on reverse phase high performance liquid chromatography with a ultraviolet detector in an isocratic mode. LC-10, Shimadzu, Japan, being operated with the provided computer software was used in this study.

\section{Results and Discussion}

The structures of studied pesticide with their IUPAC names are given in Figure 1. Pesticides are applied to fruits, vegetables and various crops at various stages of cultivation and during post harvest storage to provide protection against a range of pests, before they become available to the consumer. The formulations used have been developed with specific pesticidal properties that are dependent upon the inherent chemical and physical characteristics of constituent chemicals. Consequently, pesticides comprise a large number of substances that belong to many different chemical classes.

To ensure the safety of food for consumers and regulate international trade, legislations such as European Union directives has established maximum residue limits for pesticides in food stuff. Thorough monitoring of pesticide residues is crucial for proper risk assessment of human exposure through food (Shams-ul-Alam and Awwal Ahmed 2009). Standard chromatograms for four pesticides i.e., chlorpyrifos, cypermethrin, deltamethrin and dichlorovos are shown in Figure 2. 
The maximum pesticide residue levels analysed in apple, peach, and potato are given in Table 1. Retention time of pesticides is considered in \pm 0.10 variations and the maximum residue levels (MRLs) in the selected fruits and vegetable are calculated accordingly. The results of these pesticides are then compared with the extraneous maximum residue limits (MRLs) of these pesticides in fruits and vegetables as specified in food standards by Codex Alimentarius issued by food and agriculture organization (FAO) and world health organization (WHO).

The residual amount of chlorpyrifos (0.924 $\mu \mathrm{g} / \mathrm{g}$ ) in peach is higher than MRLs specified by Codex Alimentarius (Table 1). Chlorpyrifos is a crystalline organophosphate insecticide and was introduced in 1965 by Dow Chemical Company. It acts on the nervous system of insects by inhibiting acetylcholinesterase. The crops with the most intense chlorpyrifos use are cotton, corn, almonds, and fruit trees including oranges, bananas and apples (Muller and Franz, 2000).

Chlorpyrifos is moderately toxic to humans and chronic exposure has been linked to neurological effects, developmental disorders, and autoimmune disorders. Exposure during pregnancy retards the mental development of children, and most use in homes has been banned since 2001 in the United States (Muller and Franz, 2000).

Results (Table 1) indicate that levels for cypermethrin in apple, peach and potato are $0.875,1.630$ and $0.192 \mu \mathrm{g} / \mathrm{g}$ respectively. The residual amount of cypermethrin $(1.630 \mu \mathrm{g} / \mathrm{g})$ in peach is higher than MRLs specified by Codex Alimentarius (Table 1) and for apple and potato, these levers are found within the pescribed safe limits, however, their prolong use in the infected fruits and vegetable may result toxic health effects. Literature shows that cypermethrin, a broad-spectrum insecticide, is a synthetic pyrethroid used as an insecticide in large-scale commercial agricultural applications because it behaves as a fast-acting neurotoxin in the insects. It is easily degraded on soil and plants but can be effective for weeks when applied to indoor inert surfaces. Exposure to sunlight, water and oxygen will accelerate its decomposition.

Cypermethrin is highly toxic to fish, bees and aquatic insects. In humans, cypermethrin is deactivated by enzymatic hydrolysis to several carboxylic acid metabolites, which are eliminated in the urine (Stephenson, 1982, Pascual and Peris 1992, Baselt 2008).
Results given in Table 1 show that levels for deltamethrin in apple, peach and potato are $0.241,0.454$ and $0.065 \mu \mathrm{g} / \mathrm{g}$ respectively. Again these maximum residue levels (MRL) are within the pescribed safe limits, however, repeated use of fruits and vegetable infected with deltamethrin may result toxic health effects. Literature indicates that deltamethrin, widely used in the world, is a pyrethroid ester insecticide that kills insects through dermal contact, digestion and paralyzes the nervous system of its insect target, leading to eventual death. As deltamethrin is a neurotoxin and it temporarily attacks the nervous system of any animal with which it comes into contact. It is reported that deltamethrin, most toxic of the pyrethroids, is able to pass from a woman's skin through her blood and into her breast milk. It was first synthesized in 1974, and since has been used primarily on cotton, coffee, maize, cereals, fruits, and stored products (Muller and Franz, 2000).

As a lipophillic compound, deltamethrin is not soluble in water and therefore is highly stable in the physical environment. Unlike many pyrethroids, deltamethrin is also stable in air and sunlight: when exposed to either, it does not degrade, even after two years' time at 40 degrees Celsius (Muller and Franz, 2000).

In studies done on workers in agricultural settings reported that deltamethrin can produce a variety of acute health conditions with several ecological risks, particularly by causing algal blooms and reducing bee populations. Deltamethrin also plays key role in controlling malaria vectors, and is used in the manufacture of long-lasting insecticidal mosquito nets. Recently, in South Africa, residues of deltamethrin were found in breast milk, together with DDT, in an area that used DDT treatment for malaria control, as well as pyrethroids in small-scale agriculture.

Deltamethrin is not mobile in the environment because of its strong adsorption on particles, its insolubility in water, and very low rates of application; however, it still has risks to the ecosystem in which it is applied (Sereda and Meinhardt 2006).

Levels for dichlorvos (DDVP) in apple, peach and potato are $0.283,0.021$ and 0.014 $\mu \mathrm{g} / \mathrm{g}$ respectively (Table 1 ). These maximum residue levels (MRL) are also within the pescribed safe limits. However, repeated use of fruits and vegitable infected with dichlorvos may result toxic health effects. Literature indicates 
that dichlorvos (DDVP) is a highly volatile organophosphate (OP), widely used as an organophosphorus insecticide in developing countries since about 1955. It is effective against mushroom flies, aphids, spider mites, caterpillars, thrips, and whiteflies in greenhouse, outdoor fruit, and vegetable crops. It was studied that each 10-fold increase in urinary concentration of organophosphate metabolites was associated with a $55-72 \%$ increase in the odds of Deficit Hyperactivity Disorder (ADHD) and urinary metabolites in children aged between 8-15 years old. Because dichlorvos degrades fairly rapidly so, it is not generally found as a residue on food (Fraser et al. 1989).

Dichlorvos is absorbed through all routes of exposure and damages DNA of insects (Espeland et al. 2010). Dichlorvos can inhibit cholinesterase levels in humans which may lead to short or longer term neurotoxic effects. Since it is an acetylcholinesterase inhibitor, its overdose symptoms in humans are weakness, headache, tightness in chest, blurred vision, salivation, sweating, nausea, vomiting, diarrhea, and abdominal cramps (Maryse 2010).

\section{Conclusion and Recommendations}

Fruits and vegetables production is substantially reduced by infestation of various insects and pests. The pest infestation causes 30$40 \%$ loss to the yields and some times the loss is high. Pesticides, indispensible components of integrated pest management (IPM), can reduce these losses. However, indiscriminate applications of these pesticides to the crops results in food and food commodities with consequential hazards. The health hazards can be minimized considerably or eliminated if these residues are kept below their prescribed safe levels.

\section{References}

Abhilash, P.C., Singh, N. (2009), Pesticide use and application: An Indian scenario. J Hazard Mater, $165,1-12$.

Ambedkar, J.S., Pawar, A.S., Sakhare, M.V. (1999), Evaluation of some neem formulations against sucking pests on Okra. Proc Nat Symp. Crop Pest and Disease Management: Challenges for the Next Millennium, New Delhi, p. 63.

American Chemical Society (2009, March 24). New 'Green' Pesticides Are First To Exploit Plant Defenses In Battle Of The Fungi. ScienceDaily. Retrieved May 16, 2013, from http://www.sciencedaily.com

/releases/2009/03/090323110447.htm

Baselt, R. (2008), Disposition of Toxic Drugs and Chemicals in Man, $8^{\text {th }}$ edition, Biomedical Publications, Foster City, CA, pp. 389-391.

Cheng, C.L. and Bersamira, K.V. (1994), Pesticides: It's hazardous effects on the benguet farmers and the environment, Unique Printing Press, Baguio City, Philippines.

Espeland, M., Irestedt, M., Johanson, K.A., Åkerlund, M., Bergh, J.E. and Källersjö, M. (2010), Dichlorvos exposure impedes extraction and amplification of DNA from insects in museum collections. Frontiers in Zoology, 7: 2.

Fraser, J.P., Duncan, G. and Tomlison, J. (1989), Effects of a cholinesterase Inhibitor on salmonid lens, a possible cause for the Increased Incidence of cateract in salmon salmo salar. Exp. Eye Res. 49, 293-98.

Gilden, R.C., Huffling, K. and Sattler, B. (2010), Pesticides and health risks. J. Obstet. Gynecol. Neonatal Nurs. 39, 103-10.

Hernandez-Borges, J., Cabrera, J.C, RodriguezDelgado, M.A., Hernandez-Suarez, E.M. and Sauco, V.G. (2009), Analysis of pesticide residues in bananas harvested in the Canary Islands (Spain). Food Chemistry 113: 313-319.

Kmellara, B., Fodora, P., Parejab, L., Ferrerc, C., Martinez-Urozc, M.A., Valverdec, A. and Fernandez-Albac, A.R. (2008), Validation and uncertainty study of a comprehensive list of 160 pesticide residues in multi-class vegetables by liquid chromatography-tandem mass spectrometry, J Chromatography A, 1215: 37-50.

Maryse, F.B., David, C.B., Robert, O.W. and Marc, G.W. (2010), Attention-Deficit/Hyperactivity Disorder and Urinary Metabolites of Organophosphate Pesticides, Pediatrics 125:6 e1270-e1277

Miller, G.T. (2006), Living in the Environment: Principles, Connections, and Solutions. Publisher: Cengage Learning.

Min, L., Hashi, Y., Yuanyuan, S. and Jinming, L. (2006), Determination of carbamate and organophosphorus pesticides in fruits and vegetables using liquid chromatography-mass spectrometry with dispersive solid phase extraction. Chin J Anal Chem, 34(7), 941-945.

Muller, Franz, ed. (2000). Agrochemicals: Composition, Production, Toxicology, Applications. Toronto: Wiley-VCH. p. 541.

Ortelli, D., Edder, P. and Corvi, C. (2004), Multiresidue analysis of 74 pesticides in fruits and vegetables by liquid chromatographyelectrospray-tandem mass spectrometry, Analytica Chimica Acta, 520: 33-45. 
Pascual, J.A. and Peris, S.J. (1992), Effects of forest spraying with two application rates of cypermethrin on food supply and on breeding success of the blue tit (Parus caeruleus). Environ Toxicol Chem. 11: 1271-1280.

Sereda, B.B. and Meinhardt, H.M. (2006), Simultaneous presence of DDT and pyrethroid residues in human breast milk from a malaria endemic area in South Africa. Environ Pollution 144: 902-917.

Shams-ul-Alam, Awwal Ahmed, (2009), Status of Integrated Pest Management (IPM) in SAAR Countries, SAARC Agriculture Centre, Dhaka, Bangladesh.

a)

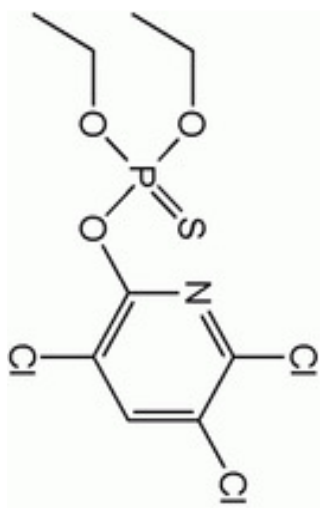

$O, O$-diethyl $O-3,5,6-$ trichloropyridin-2-yl phosphorothioate b)

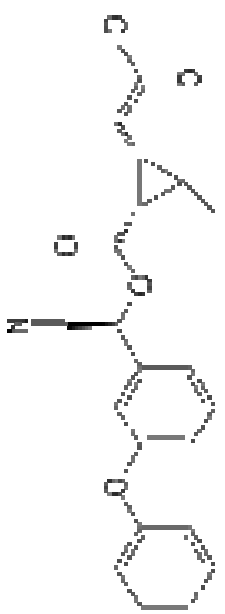

[Cyano-(3-

phenoxyphenyl)methyl]3(2,2-dichloroethenyl)-2,2dimethylcyclopropane-1carboxylate
Siddique T., Zahir, Z.A. and Frankenberger, W.T Jr. (2003), Reversed phase liquid chromatographic. Method for analysis of endosulfan and its major metabolites. J Liq Chromatogr Relat Technol 26,1069-1082.

Stephenson, R.R. (1982), Aquatic toxicology of cypermethrin. I. Acute toxicity to some freshwater fish and invertebrates in laboratory tests. Aquatic Toxicology 2 (3), 175-185.

Tariq, M.I., Afzal, S., Hussain, I. and Sultana, N. (2007), Pesticides Exposure in Pakistan: A Review, Environ Intl, 33, 1107-1122. c)

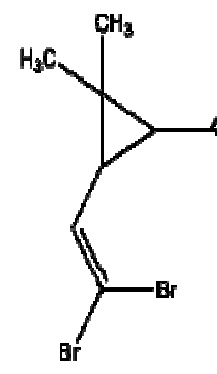

d)<smiles>[CH]C([O])=CO[Te](=O)(O[Te])O[Te]</smiles>

$2,2-$

[(S)-cyano-(3-phenoxyphenyl)methyl] $(1 R, 3 R)-3-(2,2-$ dibromoethenyl)-2,2-dimethylcyclopropane-1-carboxylate dichlorovinyl

dimethyl

phosphate

Figure 1 Chemical structures with IUPAC names of a) chlorpyrifos, b) cypermethrin, c) deltamethrin, and d) dichlorvos

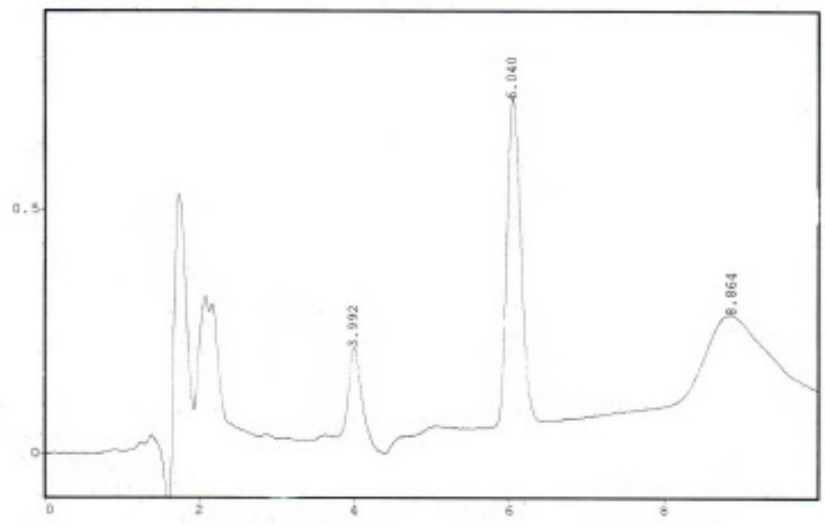

a)

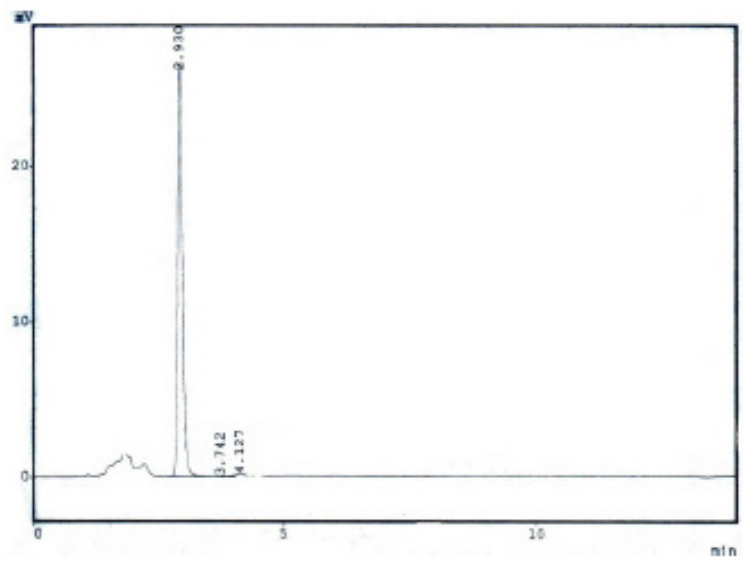

b) 


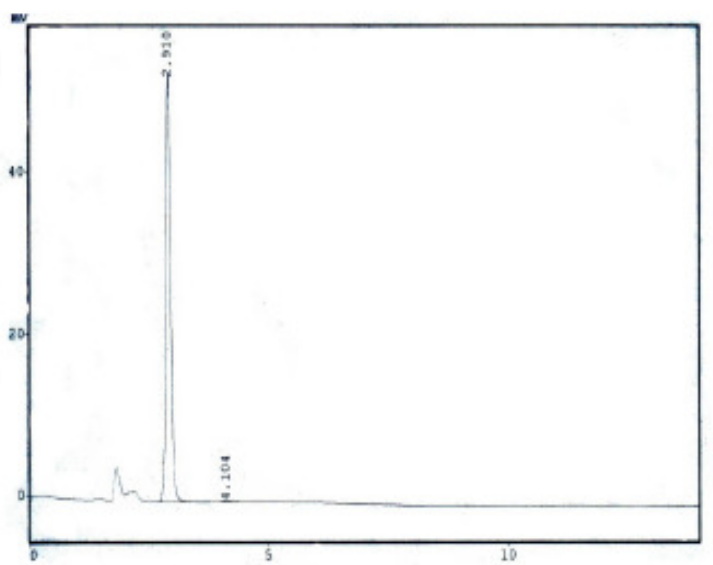

c)

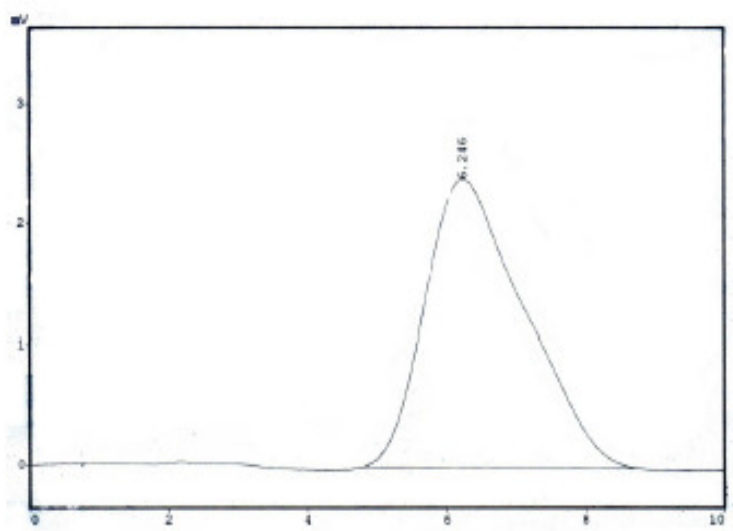

d)

Figure 2 Chromatograms of standard pesticides; a) Chlorpyrifos, b) Cypermethrin, c) Deltamethrin, and d) Dichlorovos
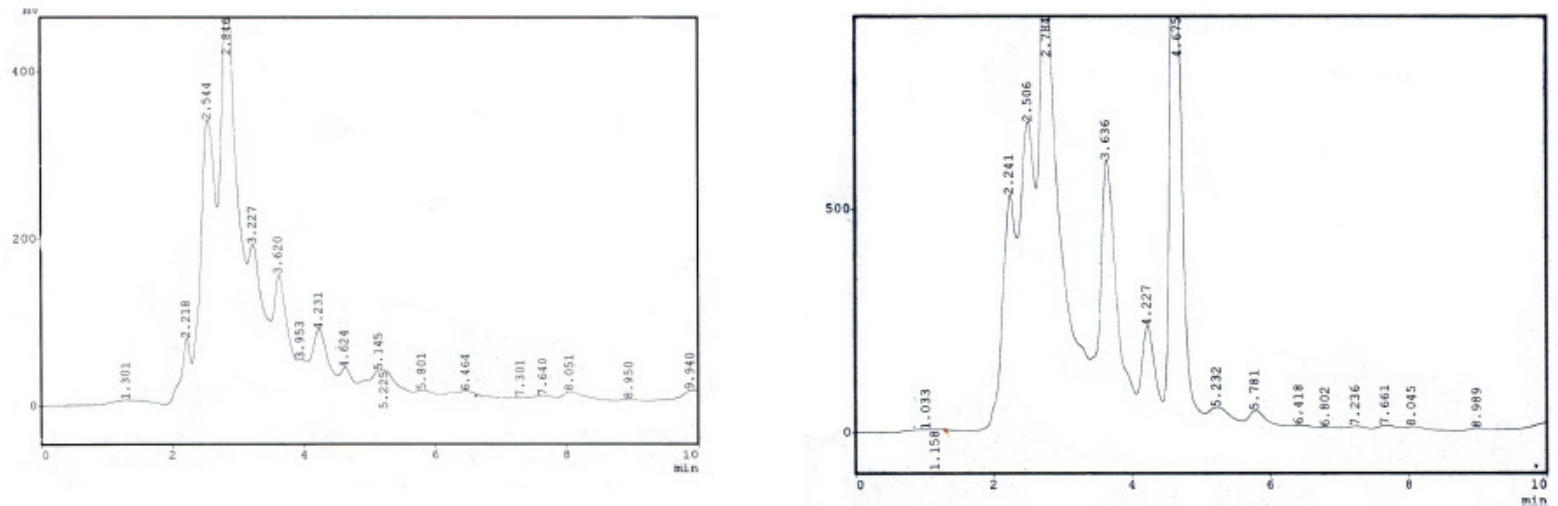

Figure 3 Chromatograms of pesticide residues from different fruits and vegetables; a) apple, b) peach, and c) potato

Table 1 Integration parameters and maximum residue levels (MRL) found for fruit and vegetable samples using RP- HPLC equipped with UV-detector

\begin{tabular}{cccc}
\hline $\begin{array}{c}\text { Name of the } \\
\text { Pesticide }\end{array}$ & $\begin{array}{c}\text { Fruit and } \\
\text { vegetable }\end{array}$ & Retention Time* & $\begin{array}{c}\text { Maximum Residue Levels } \\
(\mathrm{MRL}) \\
(\mu \mathrm{g} / \mathrm{g})\end{array}$ \\
\hline Chlorpyrifos & Apple & 2.784 & 0.860 \\
& Peach & 3.082 & 0.924 \\
Cypermethrin & Potato & 3.162 & 0.137 \\
& Apple & 2.785 & 0.875 \\
& Peach & 2.749 & 1.630 \\
Deltamethrin & Potato & 2.881 & 0.192 \\
& Apple & 2.787 & 0.241 \\
& Peach & 2.756 & 0.454 \\
Dichlorvos & Potato & 2.700 & 0.065 \\
& Apple & 6.418 & 0.283 \\
& Peach & 6.490 & 0.021 \\
& Potato & 6.076 & 0.014 \\
\hline
\end{tabular}

$*$ Retention time of pesticides is considered in \pm 0.10 variations. 